\title{
Regionale Arbeitsmärkte für höherqualifizierte Frauen und Männer in der Schweiz
}

\section{Einleitung}

Arbeitsmärkte sind in verschiedener Hinsicht tiefgreifend strukturiert. Zwei der augenfälligsten Strukturen sind - weltweit gesehen - die Geschlechtersegregation und die räumlichen Unterschiede der Arbeitsmärkte.

Der Ausdruck Geschlechtersegregation enthält eine horizontale und eine vertikale Komponente. Horizontale Geschlechtersegregation bezeichnet das soziale Phänomen, daß Frauen und Männer meist unterschiedliche Berufe ausüben und damit in hohem Maße auf separierten Arbeitsmärkten agieren. Frauen sind in wenigen Berufen konzentriert, die oft durch geringere Aufstiegschancen und niedrigere Löhne gekennzeichnet sind (EIDGENÖSSISCHE KOMMISSION FÜR FRAUENFRAGEN 1995, S. 141). Typische Frauenberufe sind zum Beispiel Krankenschwester, Sekretärin, Verkäuferin oder Kindergärtnerin.

Unabhängig von der Art des ausgeübten Berufes sind Frauen in den hierarchisch höheren Rängen der privaten und staatlichen Unternehmen überall sehr deutlich untervertreten und damit von wichtigen wirtschaftlichen, politischen und wissenschaftlichen Entscheidungsgremien weitgehend ausgeschlossen. Hierfür steht der Begriff der vertikalen Segregation. Das im Durchschnitt wesentlich tiefere Einkommen der Frauen im Vergleich zu demjenigen der Männer ist neben der eigentlichen Einkommensdiskriminierung' eine direkte Folge der vertikalen Geschlechtersegregation im Berufsleben. Unter dem Aspekt der Gleichstellung der Frauen und Männer in der Gesellschaft stellt die Geschlechtersegregation in der Erwerbstätigkeit ein zentrales gesellschaftspolitisches Problem dar.

Räumlich gesehen sind die Arbeitsmärkte auf verschiedenen Maßstabsebenen tiefgreifend segmentiert. Auf internationaler Ebene unterscheiden wir beispielsweise zwischen «Industrieländern» oder «Hochlohnländern» einerseits und «Entwicklungsländern» oder "Tieflohnländern» andererseits. In den Industrieländern weist der größte Teil der Bevölkerung eine Berufsausbildung auf und ist im Industrie- und Dienstleistungssektor tätig, während in den Entwicklungsländern nach wie vor sehr viele Menschen über keine Ausbildung verfügen und als ungelernte Arbeitskräfte und/oder in der Landwirtschaft tätig sind. Die revolutionären Entwicklungen in der Informations- und Kommunikationstechnik der letzten Jahre führten zu einer zunehmenden Vernetzung und gegenseitigen Abhängigkeit der unterschiedlichen globa- len Arbeitsmärkte (Globalisierung, neue internationale Arbeitsteilung).

Auf nationaler oder regionaler Ebene sind analoge Prozesse der funktionalräumlichen Arbeitsteilung zu beobachten. Bestimmte Wirtschaftsbereiche werden zum Beispiel aus den großen Städten in suburbane oder ländliche Standorte mit tieferen Boden- und Lohnkosten ausgelagert. Hauptsitze von Mehrbetriebsunternehmen und kommerzielle Dienstleistungen konzentrieren sich dagegen in den städtischen Zentren. Dieser wirtschaftliche Strukturwandel schlägt sich deutlich in den regionalen Arbeitsmarktstrukturen, aber auch in den regionalen Einkommensunterschieden nieder. Regionale Arbeitsmärkte stellen damit ein erstrangiges Problem einer Regionalpolitik dar, deren Ziel es ist, die Wohlstands- und Lebensqualitätsunterschiede zwischen den einzelnen Landesteilen zu verringern.

Angesichts der nach wie vor weitgehend segregierten Arbeitsmärkte für Frauen und Männer sind geschlechtermäßig undifferenzierte Aussagen über regionale Arbeitsmarktstrukturen in der Schweiz nicht wirklichkeitsgerecht. Am Beispiel der höherqualifizierten Erwerbstätigen (vgl. Kap. 3) werden im vorliegenden Beitrag zwei der angesprochenen zentralen Arbeitsmarktstrukturen, die vertikale Geschlechtersegregation und die regionale Arbeitsteilung, für die Schweiz miteinander verknüpft. Das Ziel besteht darin, wichtige Aspekte der beruflichen Realität von Männern und Frauen in den verschiedenen Landesteilen zu beschreiben und mögliche Erklärungen dafür vorzustellen. ${ }^{2}$

\section{Vertikale Geschlechtersegregation des schweizerischen Arbeitsmarktes}

\section{Sozio-professionelle Kategorien der erwerbstätigen Personen}

Als Indikator zur Messung der vertikalen Geschlechtersegregation des schweizerischen Arbeitsmarktes eignen sich die sozio-professionellen Kategorien der erwerbstäti-

Elisabeth Bühler, Dr., Guido Dorigo, Dr., Geographisches Institut der Universität Zürich, Winterthurerstraße 190, 8057 Zürich. 
gen Personen. Diese Kategorien wurden von Fachleuten des Bundesamtes für Statistik mit Hilfe folgender Angaben aus den Fragebogen der eidgenössischen Volkszählung gebildet: Art der beruflichen Tätigkeit, Stellung im Beruf, abgeschlossene Ausbildung (für genauere Angaben zum Vorgehen vgl. JOYE, SCHULER 1995).

$1990^{3}$ waren rund $39 \%$ aller erwerbstätigen Personen in der Schweiz Frauen. Wie aus Tabelle 1 hervorgeht, sind die Frauen bei den zwei sozio-professionellen Kategorien Ungelernte Arbeitskräfte und Qualifizierte nichtmanuelle Berufe übervertreten. In den hohen sozioprofessionellen Kategorien Oberstes Management und Freie Berufe sind sie dagegen sehr deutlich untervertreten. Untervertreten sind die Frauen auch in den Kategorien Andere Selbständige, Akademische Berufe und oberes Kader und Qualifizierte manuelle Berufe. ${ }^{4}$

\section{Veränderungen in den 80er Jahren}

Unter dem Gesichtspunkt des quantitativen Wachstums der Arbeitsplätze dürfen die 80er Jahre auch in der Schweiz als "Golden Eighties» bezeichnet werden. Bei einem Bevölkerungswachstum zwischen 1980 und 1990 von rund $8 \%$ betrug die Zunahme der erwerbstätigen Personen im selben Zeitraum bemerkenswerte 17\%. 55\% dieser zusätzlichen Erwerbstätigen sind Frauen, mehr als zwei Drittel davon sind teilerwerbstätige Frauen.
In anderen westeuropäischen Ländern fanden ähnliche Entwicklungen statt. Das markante Eindringen der Frauen in die Erwerbsarbeit in den 80er Jahren hat jedoch nirgendwo zu einer entsprechenden Reduktion der anderen Segregationsmerkmale geführt (BECKER 1993, S. 227). Im Gegenteil: Untersuchungen aus Großbritannien betonen zum Beispiel, daß diese "Feminisierung der Erwerbsarbeit» eng zusammenhängt mit einer Zunahme der schlecht entlöhnten, unsicheren Gelegenheitsarbeit und die geschlechterspezifische vertikale Segregation sich eher noch verstärkt hat (McDOWELL. COURT 1994, S. 1400).

Auch im "frauenfreundlichen Wohlfahrtsstaat" Schweden ist die horizontale und vertikale Geschlechtersegregation des Arbeitsmarktes heute im großen und ganzen noch dieselbe wie in den 60er Jahren (FORSBERG 1994, S. 1239). Frauen dominieren auch hier in den tiefen beruflichen Stellungen, in den monotonen Tätigkeiten der Nahrungsmittel-, Textil- und Elektronikindustrie sowie in den Reinigungs-, Pflege- und Büroberufen. Im Unterschied zu Großbritannien sind die Einkommensunterschiede zwischen Frauen und Männern in Schweden dagegen geringer, und die zeitliche Vereinbarkeit von Familie und Beruf wird dank dem umfassenden Angebot an familienexternen Kinderbetreuungseinrichtungen wesentlich erleichtert.

In der Schweiz sind in den 80er Jahren gewisse Polarisierungstendenzen in der Frauenerwerbstätigkeit eingetre-

Tabelle 1

Sozio-professionelle Kategorien der erwerbstätigen Frauen und Männer in der Schweiz 1990

\begin{tabular}{|c|c|c|c|c|c|c|}
\hline & \multicolumn{2}{|c|}{ Absolute Zahlen } & \multicolumn{2}{|c|}{$\begin{array}{c}\text { Vertikale } \\
\text { Prozentanteile' }\end{array}$} & \multicolumn{2}{|c|}{$\begin{array}{c}\text { Horizontale } \\
\text { Prozentanteile? }\end{array}$} \\
\hline & Frauen & Männer & Frauen & Männer & $\begin{array}{l}\text { Frauen- } \\
\text { anteil }\end{array}$ & $\begin{array}{l}\text { Männer- } \\
\text { anteil }\end{array}$ \\
\hline Oberstes Management & 4475 & 42605 & 0,3 & 2,1 & 9,5 & 90,5 \\
\hline Freie Berufe $^{3}$ & 4981 & 26100 & 0,4 & 1,3 & 16,0 & 84,0 \\
\hline Andere Selbständige & 70713 & 252441 & 5,5 & 12,3 & 21,9 & 78,1 \\
\hline Akademische Berufe und oberes Kader & 55563 & 238998 & 4,3 & 11,6 & 18,9 & 81,1 \\
\hline Intermediäre Berufe ${ }^{4}$ & 213548 & 444311 & 16,5 & 21,6 & 32,5 & 67,5 \\
\hline Qualifizierte nicht-manuelle Berufe & 520985 & 269979 & 40,3 & 13,1 & 65,9 & 34,1 \\
\hline Qualifizierte manuelle Berufe & 53170 & 381888 & 4,1 & 18,5 & 12,2 & 87,8 \\
\hline Ungelernte Angestellte und Arbeiter & 314971 & 322651 & 24,4 & 15,7 & 49,4 & 50,6 \\
\hline Nicht zuteilbare Erwerbstätige & 53684 & 80891 & 4,2 & 3,9 & 39,9 & 60,1 \\
\hline Total & 1292090 & 2059864 & 100,0 & 100,0 & 38,5 & 61,5 \\
\hline \multicolumn{7}{|c|}{ ' Frauen dieser Kategorie in Prozent aller Frauen (Männer analog) } \\
\hline \multicolumn{7}{|c|}{${ }^{2}$ Frauen dieser Kategorie in Prozent aller Personen in dieser Kategorie (Männer analog) } \\
\hline \multicolumn{7}{|c|}{${ }^{3}$ Selbständige Akademiker und Akademikerinnen } \\
\hline \multicolumn{7}{|c|}{${ }^{4}$ Personen im mittleren Kader sowie Personen mit einer weiterführenden Ausbildung } \\
\hline $\begin{array}{l}\text { Grundgesamtheit: Erwerbstätige Männer } \\
\text { Lehrlinge }\end{array}$ & Frauen am & Arbeitsort, d & & 6 Wocl & & \\
\hline
\end{tabular}


ten, indem eine Minderheit von Frauen es geschafft hat, in hohe berufliche Positionen vorzudringen, die große Zahl der Frauen jedoch immer noch in den unteren Hierarchiestufen tätig ist (BÜHLER 1995, CHARLES 1995). Aus diesen Ausführungen geht hervor, daß eine Zunahme der weiblichen Erwerbsbeteiligung für sich allein betrachtet nichts aussagt über eine Verbesserung der Stellung der Frauen in der Erwerbsarbeit. Hierzu sind in erster Linie Daten zur Entwicklung der vertikalen Segregation und der Einkommen notwendig. Die jüngeren Entwicklungen in Europa zeigen insgesamt, daß sowohl die starke Zunahme der Frauenerwerbstätigkeit als auch die Persistenz der horizontalen und vertikalen Geschlechtersegregation gemeinsame Merkmale auf den europäischen Arbeitsmärkten sind. Dieser gemeinsame Trend wird jedoch durch die Wirtschafts- und Sozialpolitik in den einzelnen Staaten modifiziert im Sinne einer relativen Besser- oder Schlechterstellung der Frauen.

Auf wissenschaftlicher Ebene besteht zurzeit ein Forschungsbedarf nach genaueren Aussagen zu den Zusammenhängen zwischen den politisch-institutionellen Rahmenbedingungen und der Geschlechtersegregation in der Arbeitswelt. Vergleichende internationale oder interregionale Studien stellen eine Möglichkeit dar, diesem Ziel näherzukommen (z. B. DUNCAN 1994). ${ }^{5}$

Die entscheidende Frage, die es heute zu beantworten gilt, ist: Welche Bedingungen müssen erfüllt sein, damit eine zunehmende Erwerbsbeteiligung der Frauen auch zu einem Abbau der vertikalen Geschlechtersegregation führt?

\section{Erklärungsansätze}

Erwerbsstrukturen sind das Resultat eines Ausgleichsprozesses zwischen dem Angebot an arbeitssuchenden Frauen und Männern einerseits und der Nachfrage der Unternehmen nach Arbeitskräften andererseits. Aus ökonomischen, soziologischen und psychologischen Untersuchungen wissen wir, daß die Geschlechterstrukturen in der Erwerbstätigkeit sowohl durch das unterschiedliche Verhalten der Frauen und Männer auf der Angebotsseite zustande kommen als auch durch nicht geschlechterneutrale Strukturen und Verhaltensweisen auf Unternehmensseite (u. a. BLAU, FERBER 1991, BRUNNER et al. 1992, ZINGG SCHRUPKOWSKI 1994). Frauen wählen aus sozialisationsbedingten Überlegungen andere Berufe oder Studiengänge und bilden sich im Durchschnitt weniger lange aus. Frauen sind auch eher bereit als Männer, bei einer Familiengründung eine Teilzeitstelle anzunehmen oder vorübergehend ganz aus dem Erwerbsleben auszuscheiden.

Die Nachfragestrukturen der Unternehmen sind segmentiert und in ihren Auswirkungen keineswegs geschlechterneutral (ACKER 1990). Attraktive, gut bezahlte, insbesondere aber Arbeitsstellen mit Aufstiegsmöglichkeiten sind so konzipiert, daß sie Arbeitskräfte verlangen, die vollzeitlich und ohne Unterbrüche erwerbstätig sind. ${ }^{6}$ Dazu kommt die von Erwerbstätigen in höheren beruflichen Positionen geforderte Bereitschaft zu zeitlicher und räumlicher Flexibilität (z. B. die Bereitschaft zur Leistung von Überstunden, zu Wochenendarbeit, zu mehrtägigen Geschäftsreisen usw.). Arbeitskräfte, die neben ihrer Erwerbstätigkeit auch für die Kinderbetreuung verantwortlich sind, kommen für solche Arbeitsstellen kaum in Betracht.

Die statistische Diskriminierung der Frauen durch die Unternehmen, die allen Frauen, auch den kinderlosen beispielsweise, ein unstabiles Erwerbsverhalten unterstellt und sie deshalb bei Beförderungen und Weiterbildungsmöglichkeiten eher übergeht, ist ein weiterer Grund für den geringen Anteil hochqualifizierter Frauen in der Erwerbswirtschaft.?

\section{Regionale Unterschiede der vertikalen Geschlechter- segregation bei den höherqualifizierten Erwerbstätigen in der Schweiz}

Als höherqualifizierte Erwerbstätige werden in diesem Bericht Personen bezeichnet, welche im Rahmen der Volkszählung den drei sozio-professionellen Kategorien Oberstes Management, Freie Berufe und Akademische Berufe und oberes Kader zugeordnet wurden. Diesen Personen ist gemeinsam, daß sie über eine hohe Organisationskompetenz und in den allermeisten Fällen auch über eine hohe Ausbildungskompetenz verfügen (JOYE, SCHULER 1995, S. 56). ${ }^{8}$ Das Merkmal des obersten Managements ist die leitende Funktion (Linienfunktion) innerhalb eines größeren privaten oder staatlichen Unternehmens. Die freien Berufe weisen in der Schweiz zwei Merkmale auf: Es handelt sich um selbständig erwerbstätige Personen, die über eine Hochschulausbildung verfügen (z. B. Anwälte und Anwältinnen, Ärzte und Ärztinnen). Diese zwei erstgenannten sozio-professionellen Kategorien sind zwar zahlenmäßig relativ klein (Tabelle 1), sie repräsentieren jedoch die unternehmerische Elite des Landes (JOYE, SCHULER 1995, S. 85).

Die Kategorie Akademische Berufe und oberes Kader wird vor allem von den Angestellten gebildet, die innerhalb eines Betriebes eine gewisse Führungsfunktion innehaben. Diese soziale Ressource kann auf Entscheidungsbefugnissen (z. B. Prokuristin, Prokurist) oder auf Fachwissen (z. B. Mittelschullehrer, Mittelschullehrerin) beruhen. Unter allen sozio-professionellen Kategorien scheint diese Kategorie am besten geeignet zu sein, um wirtschaftlich dynamische Regionen zu charakterisieren und gegenüber anderen abzugrenzen (JOYE. SCHULER 1995, S. 125).

Eine für diese Untersuchung geeignete Raumgliederung der Schweiz stellen die sogenannten MS-Regionen dar. Diese Gliederung der Schweiz in 106 Regionen ermöglicht einerseits eine feinere und homogenere räumliche Differenzierung als diejenige in 26 Kantone. Andererseits unterscheiden sich die MS-Regionen bezüglich so- 


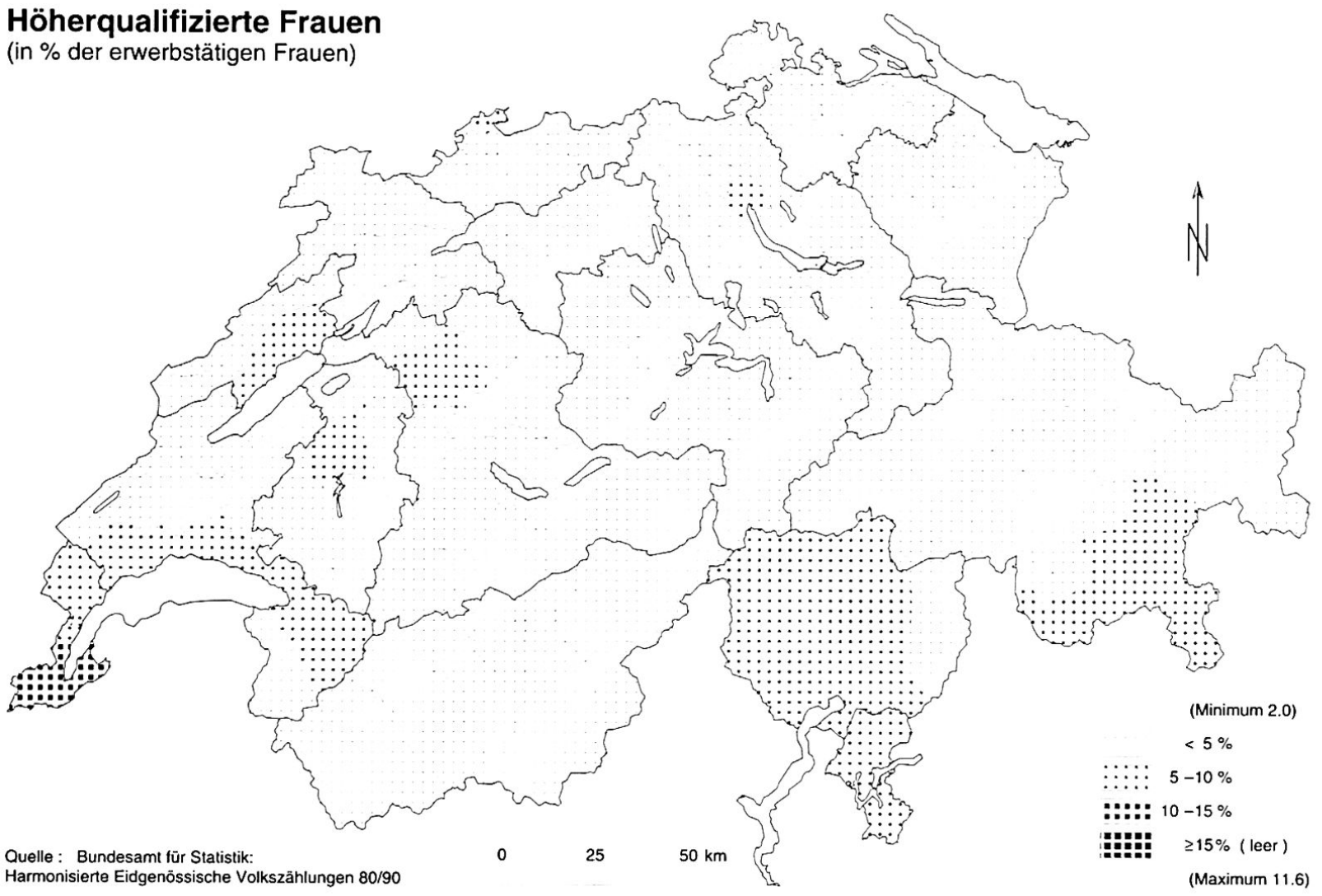

Karte 1 Höherqualifizierte Frauen in Prozent aller enwerbstätigen Frauen in der Region 1990.

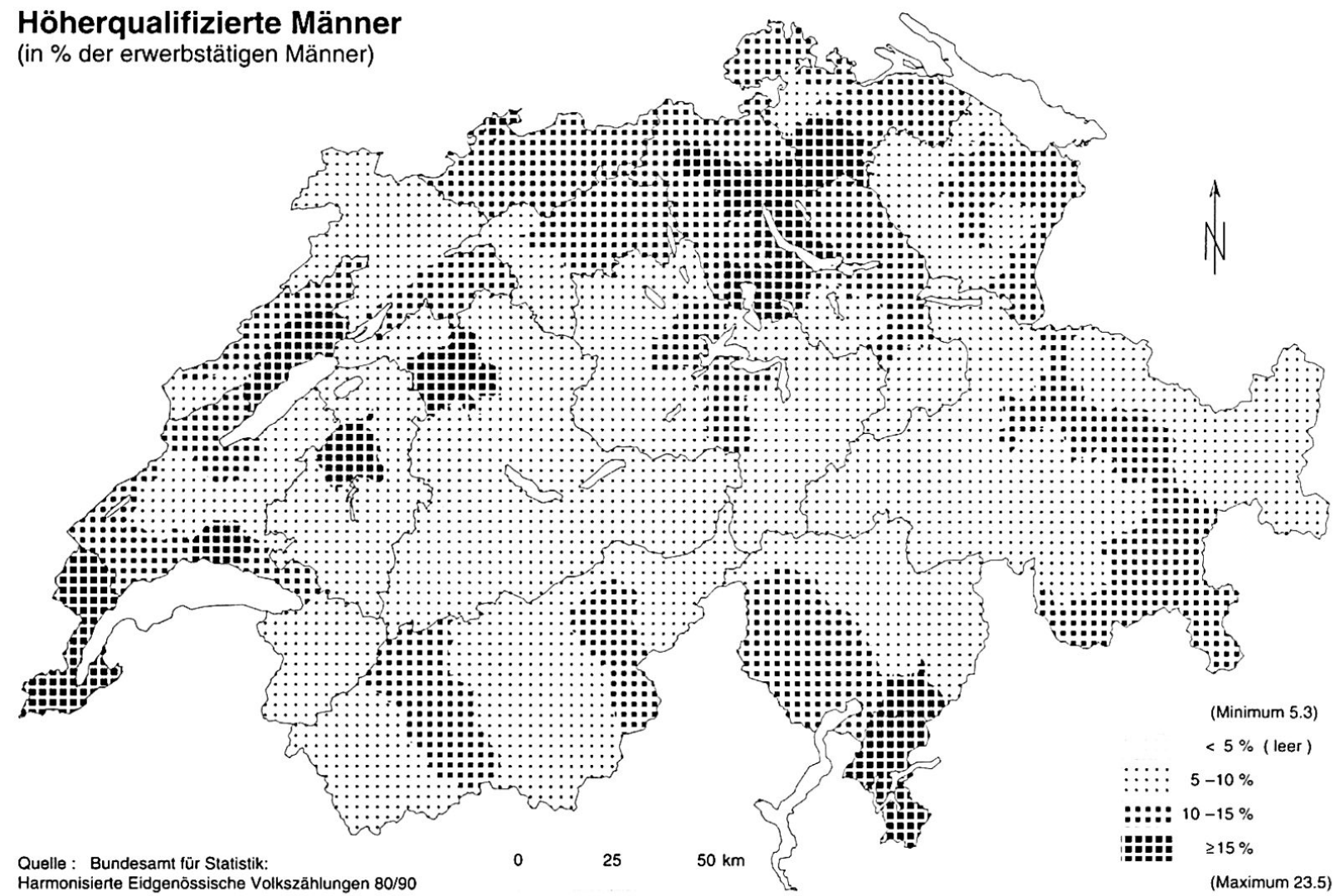

Karte 2 Höherqualifizierte Männer in Prozent aller enwerbstätigen Männer in der Region 1990. 
zialer und ökonomischer Kriterien deutlicher voneinander als die 185 Bezirke (JOYE, SCHULER 1995, S. 113). Die Raumgliederung nach MS-Regionen basiert auf dem Prinzip funktionaler Kleinregionen (vgl. BUNDESAMT FUR STATISTIK 1994a, S. 91 f.). Sie kommt deshalb in den ländlichen, klein- und mittelstädtischen Gebieten einer Regionalisierung nach Arbeitsmarktregionen am nächsten.

\section{Dominanz der hochzentralen Regionen}

Als dominierende Gruppe bei den höherqualifizierten Erwerbstätigen erweisen sich die Männer in den Großstadtzentren. Mehr als 125000 höherqualifizierte Männer arbeiteten 1990 in diesem Raumtyp. In den industriellen Mittelzentren, dem zweitgrößten regionalen Arbeitsmarkt in der Schweiz für diese Arbeitskräftekategorie, waren es gerade noch gut 46000 . Erwartungsgemäß sind die Zahlen der höherqualifizierten Frauen in allen Regionstypen viel geringer. Prozentual betrachtet sind die höherqualifizierten Frauen noch etwas stärker auf die hochzentralen Regionen konzentriert als die Männer. Allein in den 5 Großstadtzentren Zürich, Basel, Bern, Lausanne und Genf sind rund $47 \%$ der hochqualifizierten Frauen und $41 \%$ der hochqualifizierten Männer beschäftigt.
Die These "A ville plus grande, travail plus qualifié" (BAILly et al. 1990, S. 477) wird somit durch die ausgewerteten Daten der Volkszählung 1990 grundsätzlich bestätigt.

Eine Betrachtung der Karten 1 bis 3 zeigt jedoch, daß die These "A ville plus grande, travail plus qualifiè" bei einer Betrachtung der einzelnen Regionen in der Schweiz zwar recht gut auf die Männer, nicht jedoch auf die Frauen zutrifft. Die regionalen Arbeitsmärkte für die erwerbstätigen höherqualifizierten Frauen in der Schweiz weisen im Unterschied zu den Männern kein räumliches Muster auf, das im großen und ganzen der Zentrum-PeripherieDimension entspricht. Diese Besonderheiten auf den regionalen Arbeitsmärkten für hochqualifizierte Frauen in der Schweiz sollen nun etwas genauer betrachtet werden.

\section{Bessere Vertretung der hochqualifizierten Frauen im Tessin und in der Westschweiz}

Die regionalen Unterschiede und Besonderheiten im Frauenanteil an den höherqualifizierten Erwerbstätigen lassen sich grundsätzlich auf zwei verschiedene Arten darstellen. Einerseits durch die für Karte 1 berechneten Anteile der hochqualifizierten Frauen im Vergleich zu allen erwerbstätigen Frauen in der jeweiligen Region. (In Karte 2 sind analog und mit denselben Klassengrenzen

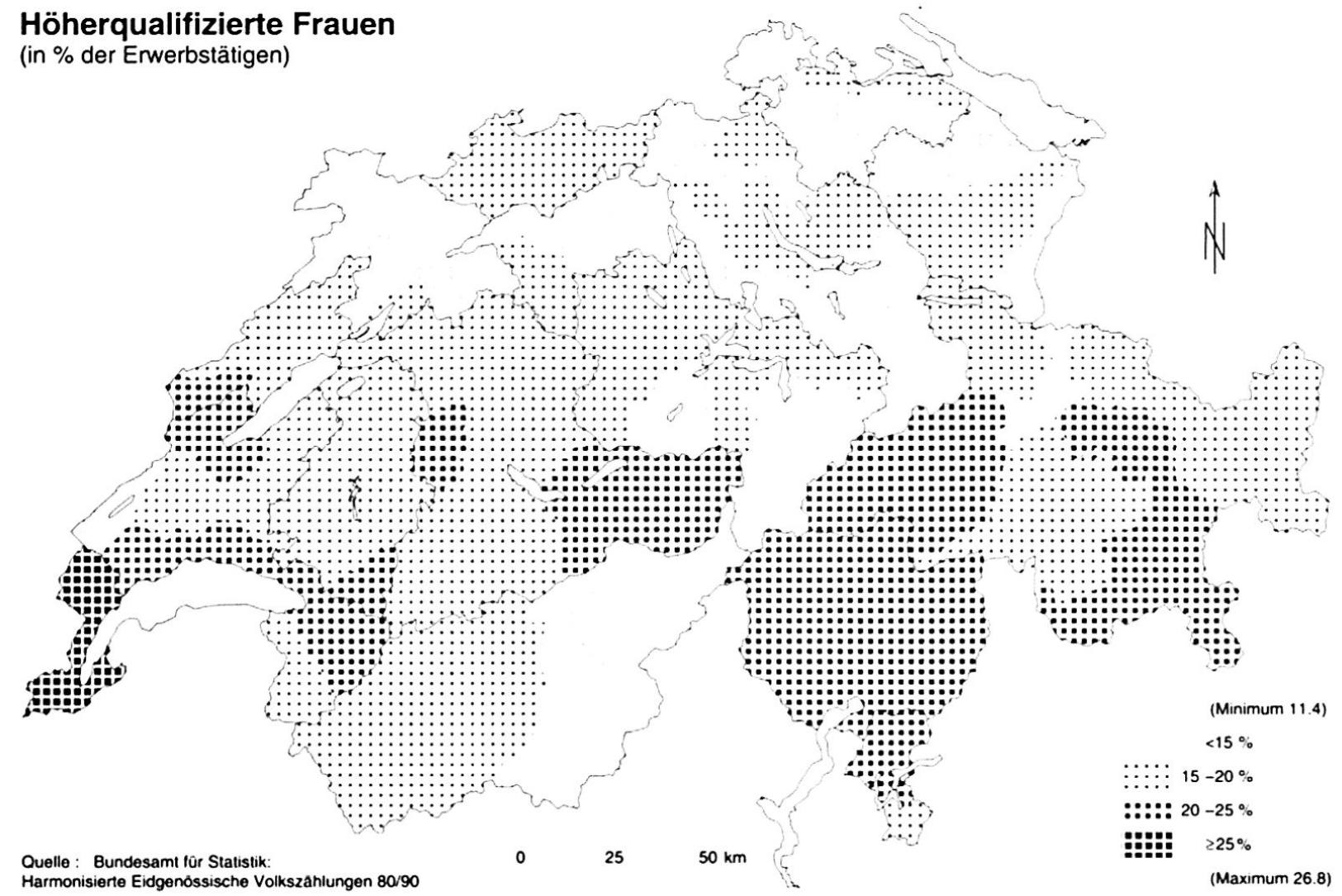

Karte 3 Höherqualifizierte Frauen in Prozent aller höherqualifizierten Erwerbstätigen in der Region 1990. 
die Männeranteile dargestellt.) Andererseits mit Hilfe der für Karte 3 berechneten Prozentanteile der hochqualifizierten Frauen an allen hochqualifizierten Erwerbstätigen (Frauen und Männer) in der Region.

Im zweiten Fall steht das Geschlechterverhältnis innerhalb der Gruppe der hochqualifizierten Erwerbstätigen im Zentrum der Betrachtung. Im ersten Fall werden die Frauen und Männer getrennt ausgewertet, dafür treten die regionalen Strukturen sehr deutlich hervor.

Erwartungsgemäß weisen alle 5 schweizerischen Großstadtzentren sowohl bei den Frauen als auch bei den Männern vergleichsweise höhere Anteile an hochqualifizierten Arbeitskräften auf (Karten 1 und 2). Bei den Frauen treten zusätzlich als Regionen mit höheren Prozentanteilen hochqualifizierter Frauen hervor: die suburbanen Regionen von Genf (Nyon, Morges/Rolle), die Mittelzentren Vevey, Neuchâtel, Fribourg, die kleinzentrale Region Aigle, die auf den Tourismus ausgerichtete Region Oberengadin sowie alle Regionen im Kanton Tessin. Keine einzige deutschschweizerische mittelzentrale, kleinzentrale oder suburbane Region erreicht jedoch einen Frauenanteil von mehr als 5,0\%.

Karte 1 zeigt somit einen deutlichen Gegensatz zwischen den deutschsprachigen und den nichtdeutschsprachigen Landesteilen. Offenbar sind die Voraussetzungen in der Westschweiz und im Tessin für die Erwerbstätigkeit von höherqualifizierten Frauen tendenziell günstiger als in der deutschsprachigen Schweiz. ${ }^{9}$

Auch die in Karte 3 dargestellten Anteile der höherqualifizierten Frauen an allen höherqualifizierten Erwerbstätigen belegen diesen Gegensatz zwischen der Deutschschweiz auf der einen Seite und der Westschweiz und dem Tessin auf der anderen Seite. Wiederum tritt das Genferseebecken als größte zusammenhängende Landesgegend mit überdurchschnittlich hohen Anteilen hochqualifizierter erwerbstätiger Frauen hervor. Mit Ausnahme der Grenzregion Mendrisio weisen auch alle Tessiner Regionen hohe Anteile hochqualifizierter Frauen auf. Weder Zürich, Basel oder Bern noch eine andere hoch- oder mittelzentrale Region der Deutschschweiz erreicht jedoch diese Werte.

\section{Bessere Vertretung der hochqualifizierten Frauen in den touristisch orientierten Regionen}

In Karte 3 fallen zusätzlich die Regionen Oberengadin, Davos, Schanfigg, Oberland Ost, Surselva und Pays d'Enhaut mit höheren Anteilen hochqualifizierter Frauen auf. In allen diesen Regionen ist der Tourismus ein wichtiger Wirtschaftszweig. Offensichtlich bieten sich heute in einem vom Tourismus geprägten regionalen Arbeitsmarkt gerade auch hochqualifizierten Frauen überdurchschnittlich viele Erwerbsmöglichkeiten.

Trotz dieser Unterschiede zwischen den regionalen "Frauenarbeitsmärkten» in der Schweiz darf eine Gemeinsamkeit im Vergleich zu den "Männerarbeitsmärkten» nicht aus dem Blick verloren werden: die starke Untervertretung der Frauen in hohen sozioprofessionellen Positionen fast überall in der Schweiz. Ein Vergleich der
Karten 1 und 2 zeigt, daß in praktisch allen Regionen die Frauenanteile um mindestens eine Klasse unterhalb der Männeranteile liegen, und aus Karte 3 geht hervor, daß in keiner Region unter den höherqualifizierten Erwerbstätigen mehr als $26,8 \%$ Frauen beschäftigt sind.

\section{Dominanz des "Goldenen (Männer-)Dreiecks»}

In Karte 2 kommt die große Dominanz des «Triangle d'Or», d. h. des Wirtschaftsraumes zwischen Basel, Zürich und Bern bzw. zwischen Basel, Olten und Winterthur (BAILLY et al. 1990, S. 481/482), sehr deutlich zum Ausdruck. Dieser Raum stellt in der Schweiz das größte zusammenhängende Gebiet von regionalen Arbeitsmärkten dar, in denen stets mehr als $10 \%$ aller Männer als hochqualifizierte Arbeitskräfte beschäftigt sind. Diese Landesgegend bietet damit für karriereorientierte Männer die attraktivsten Bedingungen an. In diesem Teil der Schweiz konzentriert sich die wirtschaftliche Entscheidungskompetenz des Landes (ROSSI, PINI 1994, S. 18/19). Im «Goldenen Dreieck» liegen u. a. sowohl die Firmenzentralen der großen Chemieunternehmen, der Großbanken, der großen Versicherungen sowie einer stattlichen Zahl großer Industrieunternehmen.

Hohe und sehr hohe vertikale Anteile hochqualifizierter Männer weist aber auch der gesamte Genferseeraum auf. Auch das Südtessin sticht als Landesgegend mit einem hohen Anteil höherqualifizierter Männer hervor (vgl. hierzu aber Fußnote 9). Sehr viele hochqualifizierte Männer arbeiten auch in der Region der Bundeshauptstadt und in derjenigen von Fribourg (La Sarine).

\section{Erklärungsansätze für die regionalen Besonderheiten der Geschlechtersegregation}

Nach BECKER (1993, S. 219) sind es vor allem drei Ursachenkomplexe, mit welchen die Unterschiede der Geschlechtersegregation zwischen verschiedenen nationalen Arbeitsmärkten erklärt werden können:

- kulturell-ideologische Faktoren,

- die Politik,

- die Struktur der Wirtschaft und wirtschaftliche Entwicklungen.

Was Becker hier für die Erklärung der Unterschiede zwischen nationalen Arbeitsmärkten als wesentlich erachtet, kann grundsätzlich auch auf die Erklärung der Unterschiede innerhalb eines Landes angewendet werden, speziell dann, wenn es - wie die Schweiz - einen ausgeprägten föderalistischen Staatsaufbau und eine kulturelle Vielfalt aufweist.

Die genauen kausalen Beziehungen zwischen diesen drei Ursachenkomplexen und den regionalen Unterschieden in der Geschlechtersegregation in der Schweiz lassen sich nur mit weiterführenden Untersuchungen ermitteln. Aus den vorhandenen statistischen Daten und der Literatur können jedoch erste Erklärungsansätze für diese regionalen Unterschiede festgehalten und Ansatzpunkte für zukünftige weiterführende Untersuchungen benannt werden. 
$\mathrm{Zu}$ den kulturellen Einflußfaktoren für die regionalen Unterschiede in der Geschlechtersegregation der Erwerbstätigen in der Schweiz gehört zweifellos der Ausbildungsbereich, insbesondere das nachobligatorische schulische Ausbildungswesen. Die Westschweiz und das Tessin weisen insgesamt markant höhere Maturitätsquoten $^{10}$ aufals die Deutschschweiz. Besonders auffällig ist in diesem Zusammenhang das Bildungsverhalten der jungen Frauen. In allen französischsprachigen Kantonen und im Tessin sind die Maturitätsquoten der Frauen höher als diejenigen der Männer, in der großen Mehrheit der deutschsprachigen Kantone ist es gerade umgekehrt, d. h., die Frauen weisen tiefere Maturitätsquoten auf als die Männer (BUNDESAMT FÜR STATISTIK 1994 b, S. 13). Auch bei den Hochschulabschlüssen sind die Frauenanteile an den französischsprachigen Hochschulen in der Schweiz deutlich höher als an den deutschschweizerischen Universitäten" (BUNDESAMT FÜR STATISTIK 1994c, S.7).

Eine erste weiterführende Auswertung der Daten der eidgenössischen Volkszählung 1990 ergab zudem, daß in den nichtdeutschsprachigen Regionen unter den höherqualifizierten Frauen deutlich mehr Frauen im Ausbildungsbereich (Wirtschaftsklasse Unterrichtswesen) beschäftigt sind als in den deutschsprachigen Regionen. Dies gilt sowohl für eine vergleichende Betrachtung der fün Großstadtzentren in der Schweiz als auch insbesondere für die weniger zentralen Regionen. In der Region Tre Valli, einer Region des Typs «industrielle Peripherie» im Tessin, arbeiten sogar $61 \%$ aller nicht selbständigen höherqualifizierten Frauen im Unterrichtswesen.

In der Schweiz gibt es große Unterschiede zwischen den Kantonen und Sprachregionen im Institutionalisierungsgrad und im Ausbaustandard der familienexternen Kinderbetreuung. Durch das großzügige Tessiner Kindergartensystem und durch die in Kindergarten und Schule praktizierten Blockzeiten ist für Tessiner Eltern beispielsweise ein wesentlich größerer (zeitlicher) Spielraum vorhanden als für Eltern in den Deutschschweizer Kantonen (EIDGENÖSSISCHE KOMMISSION FÜR FRAUENFRAGEN 1992, S. IV). Zweifellos hat dieser politisch-institutionelle Ursachenkomplex auch einen Einfluß auf die Geschlechtersegregation in der Erwerbstätigkeit.

Schließlich hat auch die wirtschaftiche Ausrichtung der Regionen auf bestimmte Wirtschaftsbranchen einen Einfluß auf die Geschlechtersegregation. Wie aus Karte 3 hervorgeht, bestehen offenbar in den touristisch ausgerichteten Regionen relativ bessere Beschäftigungsmöglichkeiten für höherqualifizierte Frauen als in vielen anderen Regionen. Eine erste zusätzliche Datenauswertung ergab, daß in den vom Tourismus geprägten Regionen sehr viele höherqualifizierte Frauen als Geschäftsführerinnen und Direktorinnen im Gastgewerbe tätig sind.

Für die Ermittlung der genauen kausalen Beziehungen zwischen diesen kulturellen, politisch-institutionellen und wirtschaftlichen Einflußfaktoren und dem Anteil höherqualifizierter Frauen auf den regionalen Arbeits- märkten in der Schweiz müssen jedoch zusätzliche statistische und qualitative Untersuchungen durchgeführt werden.

Im Vergleich zur männlichen Norm der ununterbrochenen Vollerwerbstätigkeit, verbunden mit einem geringen Arbeitsaufwand für unbezahlte Haus- und Kindererziehungsarbeit, sind die heute von Frauen ausgeübten Kombinationen von Erwerbs- und Familienarbeit enorm vielfältig. Sie reichen vom sogenannten «Drei-Phasen-Modell» ${ }^{12}$ über verschiedene Formen von Teilzeitarbeit, abwechslungsweiser Vollerwerbstätigkeit und reiner Hausfrauentätigkeit bis zu ununterbrochener Vollerwerbstätigkeit. Dazu kommt der Einfluß des Staates, der mit seinen institutionellen Regelungen und Angeboten starken Einfluß auf die Bedingungen ausüben kann, unter denen Familienarbeit und Erwerbsarbeit kombiniert wird. In der föderalistischen Schweiz ist auch diese Rolle des Staates sehr unterschiedlich.

Alle diese Faktoren führen dazu, daß die bisherigen Erkenntnisse aus geschlechtermäßig undifferenzierten regionalen Arbeitsmarktstudien nicht einfach auf Analysen regionaler Arbeitsmärkte übertragen werden können, in denen nach Frauen und Männern differenziert wird. Aufgrund der Ergebnisse dieser Studie ist davon auszugehen, daß kulturelle und politische Einflußfaktoren im Vergleich zu rein wirtschaftlichen Einflußfaktoren bei der Analyse von regionalen Unterschieden in der Frauenerwerbstätigkeit eine vergleichsweise größere Rolle spielen als in den meisten bisherigen regionalen Arbeitsmarktanalysen, die sich zumindest implizit an der männlichen Norm orientieren.

Die Resultate von weiteren Untersuchungen, in denen das Geschlechterverhältnis und die Frauenarbeit auf den verschiedenen regionalen Arbeitsmärkten in der Schweiz im Zentrum stehen, könnten insbesondere auch dazu dienen, die positiven, «frauenfördernden» Strukturen in gewissen kulturellen und politischen Kontexten und in bestimmten Wirtschaftsbranchen auch auf andere Landesgegenden oder Wirtschaftsbranchen zu übertragen und damit einen Beitrag zur Gleichstellung von Frauen und Männern leisten.

\section{Anmerkungen}

1 Geringere Entlöhnung gleichwertiger Arbeit.

2 Dieser Artikel beruht auf einer Untersuchung, die von E. Bühler im Rahmen des wissenschaftlichen Analyseprogramms der eidgenössischen Volkszählung durchgeführt wurde. G. Dorigo zeichnet verantwortlich für die Datenaufbereitung und die Gestaltung der Karten. Unter dem Titel "Regionale Arbeitsmärkte für Frauen und Männer 1980 und 1990 - Gemeinsamkeiten und Unterschiede in der vertikalen Geschlechtersegregation" wird diese Untersuchung Ende 1995 vom Bundesamt für Statistik veröffentlicht.

${ }^{3}$ Alle statistischen Angaben in diesem Beitrag basieren auf den eidgenössischen Volkszählungen 1980 und 1990. Die Zahlen beziehen sich auf folgende Grundgesamtheit: Er- 
werbstätige Frauen und Männer am Arbeitsort, die mindestens 6 Std./Woche erwerbstätig sind, ohne Lehrlinge (bei unbekanntem Arbeitsort [4\% aller Personen] wurde der Wohnort als Arbeitsort gesetzt).

${ }^{4}$ Die unterschiedlichen Frauenanteile in den qualifizierten manuellen und den qualifizierten nicht-manuellen Berufen sind in erster Linie als ein Merkmal der horizontalen Geschlechtersegregation zu interpretieren.

${ }^{5}$ Die Autorin dieser Studie ist Mitglied des «European Network on Gender Inequalities and the European Regions", das von der European Science Foundation (ESF) unterstützt wird. Ziel dieses Netzwerkes ist unter anderem, durch vergleichende länder- und regionenübergreifende Studien das Verständnis für die Zusammenhänge zwischen den kulturellen, den politisch-institutionellen und den wirtschaftlichen Rahmenbedingungen und der Geschlechterungleichheit zu verbessern.

${ }^{6}$ Diese Ausführungen beruhen auf den segmentationstheoretischen Arbeitsmarkttheorien (vgl. z.B. BRUNNER et al. 1992).

${ }^{7}$ Für weiterführende Überlegungen zu den Hintergründen der Geschlechtersegregation im Berufsleben vgl. BÜHLER 1995.

${ }^{8}$ Die Erwerbstätigen der sozio-professionellen Kategorie "Andere Selbständige" (selbständige Landwirte, Handwerker und Gewerbetreibende) verfügen zwar im Hinblick auf die Organisation ihrer eigenen Arbeit ebenfalls über eine hohe Organisationskompetenz. Ihre Ausbildungskompetenz kann jedoch sehr unterschiedlich sein und von einem lediglich obligatorischen Schulbesuch über eine Berufslehre bis zum Abschluss einer höheren technischen Lehranstalt reichen. Wegen ihrer Heterogenität wird von einer näheren Auswertung dieser sozio-professionellen Kategorie abgesehen. Für detaillierte Angaben zur Definition der sozio-professionellen Gruppen vgl. JOYE, SCHULER 1995, S. 64 f.

${ }^{9}$ Es ist anzunehmen, daß die hohen Grenzgängerinnenzahlen in gewissen Tessiner Regionen und in Genf die ausgewiesenen vertikalen Prozentwerte beeinflussen. Der genaue Umfang und die Richtung können hier jedoch nicht ermittelt werden. Bei durchschnittlich geringer qualifizierten Grenzgängerinnen würden die hier ausgewiesenen Werte tendenziell zu hoch sein. Für die Regionen des Südtessins trifft diese Einschränkung mit großer Wahrscheinlichkeit zu, da im Südtessin sehr viele gering ausgebildete Grenzgängerinnen in der Bekleidungsindustrie beschäftigt sind.

${ }^{10}$ Maturitätsquote: Anzahl der erteilten Maturitäten in Prozent der 19jährigen Wohnbevölkerung (BUNDESAMT FÜR STATISTIK 1994b, S.13)

" Französischsprachige Universitäten inkl. Fribourg: Frauenanteil an den Lizentiaten: 44\%, Deutschschweizer Hochschulen: 32\% (BUNDESAMT FÜR STATISTIK 1994 c, S. 7).

${ }^{12}$ Vollerwerbstätigkeit nach der Ausbildung, Ausstieg aus der Erwerbsarbeit bei Familiengründung, Wiedereinstieg zu einem späteren Zeitpunkt.

\section{Bibliographie}

ACKER, J. (1990): Hierarchies, jobs, bodies: a theory of gendered organisation. In: Gender \& Society, Vol. 4, Nr. 2 , S.139-158.

BAILLY, A.-S., et al. (1990): Le développement économique territorial - Lieux foyers et lieux clés. Dans: Racine, J.-B., Raffestin, C. (Ed.): Nouvelle Géographie de la Suisse et des Suisses. Lausanne, S. 477-498.

BECKER, U. (1993): Frauenerwerbstätigkeit im internationalen Vergleich. In: GRÖZINGER et al. (Hg.): Jenseits von Diskriminierung, zu den institutionellen Bedingungen weiblicher Arbeit in Beruf und Familie. Marburg, S. 205-236.

BLAU, F. D., FERBER, M. A. (1991): The Economics of Women, Men and Work. 2nd edition, Prentice-Hall.

BRUNNER, J., et al. (1992): Wirtschaftliche Chancengleichheit oder formelle Gleichstellung? WWZ-Studie Nr. 36, Wirtschaftswissenschaftliches Zentrum der Universität Basel, Basel.

BÜHLER, E. (1995): Regionale Arbeitsmärkte für Frauen und Männer 1980 und 1990 - Gemeinsamkeiten und Unterschiede in der vertikalen Geschlechtersegregation. Bundesamt für Statistik, Bern (im Druck).

BUNDESAMT FÜRSTATISTIK(1994 a): Die Raumgliederungen der Schweiz. Bern.

BUNDESAMT FÜR STATISTIK (1994 b): Maturitäten 1993. Bern.

BUNDESAMT FÜR STATISTIK (1994c): Hochschulabschlüsse 1993. Bern.

CHARLES, M. (1995): Berufliche Gleichstellung - ein Mythos? Bundesamt für Statistik, Bern (im Druck).

DUNCAN, S. (1994): Theorising differences in patriarchy. In: Environment and Planning A, 26 (8), London, S.1177-1194.

EIDGENÖSSISCHE KOMMISSION FÜR FRAUENFRAGEN (1995): Viel erreicht - wenig verändert? Zur Situation der Frauen in der Schweiz. Bern.

FORSBERG, G. (1994): Occupational sex segregation in a "woman-friendly" society - the case of Sweden. In: Environment and Planning A, 26 (8), London, S.1235-1256.

JOYE, D., SCHULER, M. (1995): Sozialstruktur der Schweiz. Sozio-professionelle Kategorien. Bundesamt für Statistik, Bern.

McDOWELL, L., COURT, G. (1994): Gender divisions of labour in the post-Fordist economy: the maintenance of occupational sex segregation in the financial services sector. In: Environment and Planning A, 26 (9), London, S.1397-1418.

ROSSI, A., PINI, G. (1994): L'entreprise multiétablissements en Suisse: sa répartition sur le territoire national et son développement dans le temps. Zürich, Manuskript.

ZINGG SCHRUPKOWSKI, T.(1994): Geschlechtsspezifische Lohndifferenzen. Schriftenreihe des Instituts für empirische Wirtschaftsforschung, Band 31, Winterthur. 